\title{
Intracranial Artery Stenosis or Occlusion Predicts Ischemic Recurrence after Transient Ischemic Attack
}

\author{
G. Ssi-Yan-Kai, N. Nasr, A. Faury, I. Catalaa, C. Cognard, V. Larrue, and F. Bonneville
}

\begin{abstract}
BACKGROUND AND PURPOSE: Patterns of DWI findings that predict recurrent ischemic events after TIA are well-established, but similar assessments of intracranial MRA findings are not available. We sought to determine the imaging characteristics of MRA that are predictive of early recurrent stroke/TIA in patients with TIA.
\end{abstract}

MATERIALS AND METHODS: We performed a retrospective analysis of 129 consecutive patients with a clinical diagnosis of TIA in whom MR imaging was done within 24 hours of symptom onset. We calculated the sensitivity, specificity, positive predictive value, and negative predictive value of $>50 \%$ stenosis or occlusion of symptomatic intracranial arteries for recurrent stroke/TIA at 7 days after TIA. We used logistic regression analysis to adjust for the clinical $A B C D^{2}$ score. We performed this analysis for symptomatic steno-occlusive lesions at any site and symptomatic steno-occlusive lesions on proximal large intracranial arteries (internal carotid artery, vertebral artery, basilar artery, and circle of Willis).

RESULTS: Forty-two (32.5\%) patients had acute ischemic lesions on DWI; 16 (12.4\%) had significant MRA lesions, of which 11 (8.5\%) were on proximal vessels. Nine patients had early recurrence (TIA, 7; minor stroke, 2). Only patients with proximal MRA lesions were at higher risk of early recurrence independent of the $A B C D^{2}$ score (adjusted odds ratio, 5.5; 95\% confidence interval, 1.1-27.8; $P=.04$ ).

CONCLUSIONS: Proximal lesions of cerebral arteries seen on MRA were predictive of recurrent stroke/TIA at 7 days. These findings suggest that MRA could be used to improve the selection of patients with TIA at high risk of early recurrent stroke/TIA.

ABBREVIATIONS: $A B C D^{2}=$ age, blood pressure, clinical deficit, duration, diabetes; EKG = electrocardiogram; $\mathrm{MIP}=$ maximum intensity projection; TOF = time of flight

$\mathbf{T}$ IA is associated with high early risk of stroke. This risk is approximately $5 \%$ at 7 days, with the greatest risk occurring in the first 48 hours. ${ }^{1-3}$ Urgent evaluation and management of patients in a dedicated TIA unit can reduce early risk of stroke. ${ }^{1}$ The clinical $\mathrm{ABCD}^{2}$ score helps identify patients at high risk. ${ }^{4,5}$

Acute MR imaging abnormalities are associated with early risk of stroke after TIA. ${ }^{6}$ DWI lesions appear to improve accurate identification of patients at high risk of stroke. ${ }^{7}$ The risk of stroke is further enhanced in the presence of intracranial artery occlusion on MRA. ${ }^{8}$ However, it is unknown whether stenotic nonoc-

Received January 26, 2012; accepted after revision March 11.

From the Departments of Neuroradiology (G.S.-Y.-K., A.F., I.C., C.C., F.B.) and Vascular Neurology (N.N., V.L.), Unité médicale de recherche U1048, Institut national de santé et de recherche médicale (N.N., V.L.), and Unité médicale de recherche U825, Institut national de santé et de recherche médicale (F.B.), University of Toulouse, Toulouse. France.

Please address correspondence to Fabrice Bonneville, MD, PhD, Department of Neuroradiology and Unité médicale de recherche U825, Institut national de santé et de recherche médicale, University of Toulouse, Toulouse, France; e-mail: bonneville.f@chu-toulouse.fr

http://dx.doi.org/10.3174/ajnr.A3144 clusive lesions on MRA are also associated with early risk of stroke. ${ }^{9}$ In addition, the effect of lesion location within the intracranial arterial tree has not been evaluated. In the present study, we sought to determine the imaging characteristics of MRA associated with increased risk of early recurrent stroke/TIA in patients with TIA.

\section{MATERIALS AND METHODS}

We performed a retrospective analysis of 129 patients consecutively admitted to the TIA clinic of our institution from June 2009 to January 2011. Patients were included in the present analysis if they had transient neurologic dysfunction caused by focal brain ischemia with or without evidence of acute infarction on DWI. ${ }^{10}$ We also included patients with persisting minor clinical signs as defined by an NIHSS score of $\leq 2$ on admission. ${ }^{11}$ Patients with isolated amaurosis fugax or diagnoses other than TIA at examination were excluded. All patients were evaluated by the same senior vascular neurologist (N.N.).

The initial work-up for patients admitted to the TIA clinic includes MR imaging, intracranial 3D TOF MRA, duplex carotid 
Table 1: Characteristics of patients stratified by MRA findings ${ }^{a}$

\begin{tabular}{lccc}
\hline & $\begin{array}{c}\text { Intracranial Artery } \\
\text { Stenosis/Occlusion } \\
(\boldsymbol{n}=16)\end{array}$ & $\begin{array}{c}\text { No Intracranial Artery } \\
\text { Stenosis/Occlusion } \\
(\boldsymbol{n}=113)\end{array}$ & $\boldsymbol{P}$ Value \\
\hline Age (yr) (mean) (SD) & $67.9(8.9)$ & $62.9(15.9)$ & .22 \\
Male & $8(50.0)$ & $66(58.4)$ & .59 \\
Hypertension & $9(56.3)$ & $48(42.5)$ & .42 \\
Cigarette smoking & $3(18.8)$ & $29(27.5)$ & 1 \\
Hypercholesterolemia & $6(37.5)$ & $42(37.2)$ & .97 \\
Previous stroke/TIA & $2(12.5)$ & $15(13.3)$ & 1 \\
Diabetes & $4(25.0)$ & $10(8.8)$ & .07 \\
ABCD ${ }^{2}$ score $\geq 4$ & $11(68.8)$ & $56(49.6)$ & .18 \\
Extracranial carotid stenosis $>50 \%$ & $2(12.5)$ & $10(8.3)$ & .64 \\
Atrial fibrillation & $1(6.3)$ & $8(7.1)$ & 1 \\
Acute infarction on DWI & $13(81.2)$ & $29(25.6)$ & $<.001$ \\
\hline
\end{tabular}

${ }^{a}$ Values in parentheses are percentages unless otherwise indicated.

Table 2: Individual data from patients with early recurrence

\begin{tabular}{llcll}
$\begin{array}{c}\text { Patient } \\
\text { No. }\end{array}$ & $\begin{array}{c}\text { Type of } \\
\text { Recurrence }\end{array}$ & $\begin{array}{c}\text { ABCD } \\
\text { Score }\end{array}$ & $\begin{array}{c}\text { Acute } \\
\text { Infarction } \\
\text { on DWI }\end{array}$ & \multicolumn{1}{c}{ MRA Lesion } \\
\hline 1 & Stroke & 4 & No & None \\
2 & TIA & 4 & Yes & Proximal occlusion \\
3 & TIA & 3 & No & Proximal stenosis \\
4 & TIA & 4 & No & None \\
5 & TIA & 5 & Yes & None \\
6 & Stroke & 6 & No & None \\
7 & TIA & 5 & No & None \\
8 & TIA & 5 & No & None \\
9 & TIA & 4 & Yes & Proximal stenosis \\
\hline
\end{tabular}

imaging, EKG, and routine blood studies. To be included in the present study, patients had to undergo MR imaging with a TOF technique within 24 hours of symptom onset. The study was approved by the medical institutional review board and local ethics committee.

\section{MR Imaging Examination}

MR imaging was performed with two 1.5T MR imaging systems (Gyroscan NT Intera; Philips Healthcare, Best, the Netherlands and Magnetom Avanto; Siemens, Erlangen, Germany). Fast protocol included axial FLAIR-weighted images, diffusion-weighted images $\left(b=0-1000 \mathrm{~mm}^{2} / \mathrm{s}\right)$, and 3D TOF images of the circle of Willis. ADC maps were constructed by linear least-squares fit on a pixel-by-pixel basis. MR angiograms were obtained by using the following imaging parameters: $25 / 7 \mathrm{~ms}$ (TR/TE), flip angle of $25^{\circ}$, five slabs, effective section thickness of $0.8 \mathrm{~mm}$, FOV of $180 \mathrm{~mm}$, and matrix of $256 \times 202$. The angiographic images were reconstructed with an MIP algorithm. Two sets of 15 MIP images were obtained by rotation of the stacked images along the horizontal and vertical axes.

Evaluation of intracranial vasculature included anterior, middle, and posterior cerebral arteries; carotid siphon; and basilar arteries. We defined proximal vessels as the following: the entire M1 (trunk of the MCA), A1 (precommunicating segment of the anterior cerebral artery), P1 (precommunicating segment of the posterior cerebral artery), V4 (intracranial vertebral artery) segments, the distal ICA, and the basilar artery. The degree of cerebral artery stenosis was assessed visually according a 3-grade scale: grade $0(0 \%-50 \%$ of vascular diameter), grade 1 (51\%-99\% of the vascular diameter or a significant stenosis with residual flow signal downstream), and grade 2 (occlusion or a vascular amputation without residual signal flow downstream). Arterial stenoses were graded by comparing the diameter of the maximally stenosed artery with the diameter of the more proximal normal segment of the same vessel. ${ }^{12}$

At admission, consultant neuroradiologists (A.F., I.C.) first evaluated these lesions. Then for this study, images were analyzed retrospectively and independently by a second neuroradiologist (G.S.-Y.-K.), who was blinded to the clinical features. In case of doubt or discordance, the final staging of stenosis was determined by an experienced senior neuroradiologist (F.B.), also blinded to clinical data.

A significant MRA lesion was defined as a stenosis of $>50 \%$ or occlusion (grade 1 or 2, respectively). Retrospectively, all significant stenoses were identified as symptomatic if they supplied the infarcted area, as observed on DWI. When no cerebral ischemic lesion was found on this latter sequence, a stenosis or an occlusion was judged as symptomatic if clinical symptoms could be related to the downstream brain parenchyma.

\section{Clinical Data}

We collected the following baseline clinical data: sex, age, blood pressure, clinical impairment and other neurologic abnormalities, duration of symptoms, and diabetes mellitus (items that contribute to the $\mathrm{ABCD}^{2}$ score); cigarette smoking; hypercholesterolemia; and duration since the first clinical symptom. Hypertension was defined as systolic blood pressure of $\geq 140 \mathrm{~mm}$ $\mathrm{Hg}$, diastolic blood pressure of $\geq 90 \mathrm{~mm} \mathrm{Hg}$, or current use of antihypertensive medication. Diabetes mellitus was diagnosed when fasting blood glucose was $\geq 126 \mathrm{mg} / \mathrm{dL}$ or there was current use of antidiabetic medication. Atrial fibrillation was characterized as a history of current electrocardiographically documented intermittent or persistent atrial fibrillation on an EKG. We calculated the $\mathrm{ABCD}^{2}$ score by using the threshold of 44 to classify patients at low risk (scores, $0-3$ ) or moderate-high risk of stroke (scores, $\geq 4)$. $^{3}$

\section{Process of Care}

After initial evaluation, patients were discharged unless they fulfilled predefined criteria for hospitalization in the stroke unit: $\mathrm{ABCD}^{2}$ score $\geq 4$, acute abnormal findings on DWI, symptomatic intracranial stenosis or occlusion on MRA, extracranial carotid artery stenosis $>50 \%$ on carotid duplex imaging, and intermittent or persistent atrial fibrillation.

Patients with proximal intracranial artery stenosis or occlusion considered symptomatic after neurologic examination were treated with aspirin combined with clopidogrel. Patients with symptomatic extracranial carotid stenosis were screened for microembolic signals by using transcranial Doppler continuous monitoring of the symptomatic middle cerebral artery. ${ }^{13}$ Patients with embolic signals were treated with aspirin combined with 
Table 3: Performance of MRI variables as predictors of recurrent transient ischemic attack or stroke at 7 days among 129 patients evaluated within 24 hours of symptom onset

\begin{tabular}{|c|c|c|c|c|c|c|}
\hline & $\begin{array}{c}\text { Sensitivity } \\
\text { (\%) (No.) }\end{array}$ & $\begin{array}{c}\text { Specificity } \\
\text { (\%) (No.) }\end{array}$ & $\begin{array}{c}\text { Positive } \\
\text { Predictive Value } \\
\text { (\%) (No.) }\end{array}$ & $\begin{array}{c}\text { Negative } \\
\text { Predictive Value } \\
\text { (\%) (No.) }\end{array}$ & $\begin{array}{c}\text { Odds Ratio } \\
\text { (95\% Confidence } \\
\text { Interval) }\end{array}$ & $P$ Value \\
\hline DWI & $33.3(3 / 9)$ & $67.5(81 / 120)$ & $7.1(3 / 42)$ & $93.1(81 / 87)$ & $1.03(0.24-4.37)$ & 1 \\
\hline MRA & $33.3(3 / 9)$ & $89.2(107 / 120)$ & $18.8(3 / 16)$ & $94.7(107 / 113)$ & $4.11(0.91-18.4)$ & .08 \\
\hline MRA proximal & $33.3(3 / 9)$ & $93.3(112 / 120)$ & $27.3(3 / 11)$ & $94.9(112 / 118)$ & $7.0(1.47-33.3)$ & .03 \\
\hline
\end{tabular}

Note:-DWI indicates acute infarction on DWI; MRA, distal or proximal stenosis or occlusion of symptomatic intracranial artery on MR angiography; MRA proximal, proximal stenosis or occlusion of symptomatic intracranial artery on MR angiography.
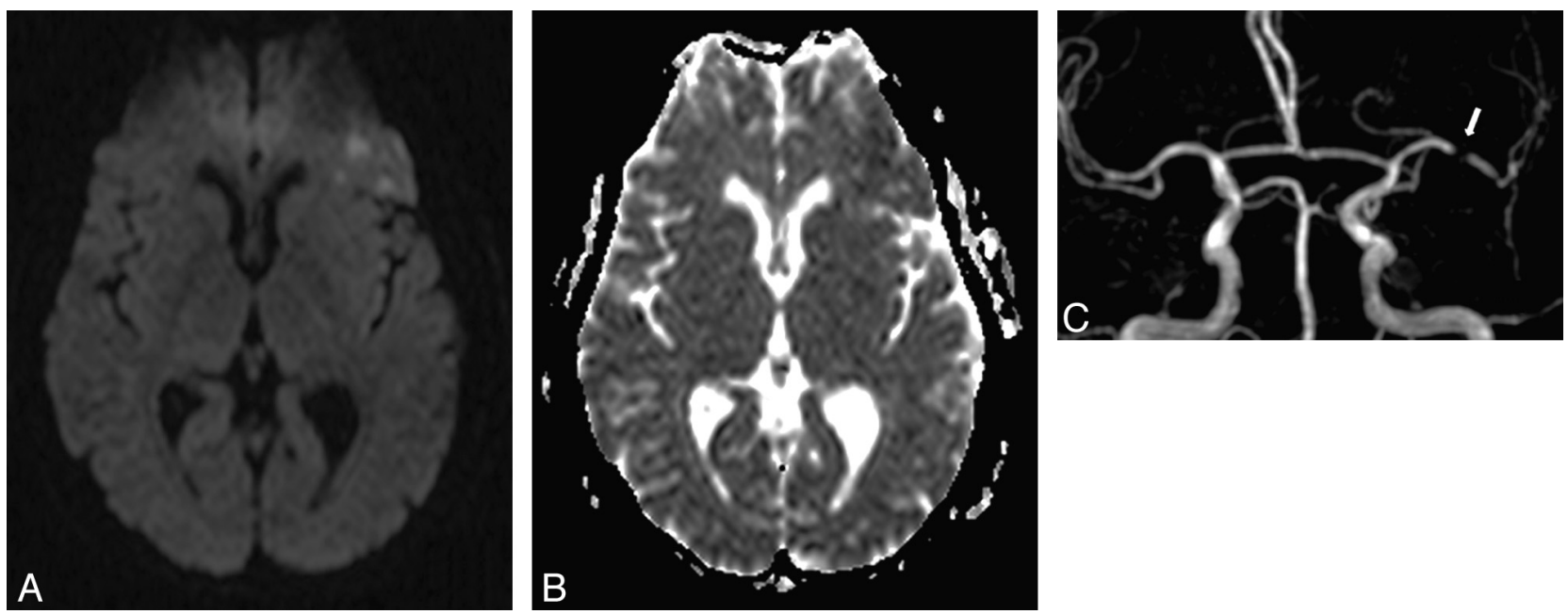

FIG 1. A 57-year-old woman with right transient hemiparesis (clinical duration $=2$ minutes, delay since the first clinical symptom $=12$ hours) $A B C D^{2}$ score $=4$. A, DWI shows some foci of high signal intensity in the left frontal lobe with a decrease of ADC on the ADC map (B). C, 3D TOF reveals a significant proximal stenosis of the left Ml segment (arrow). The patient had another right transient impairment in the subsequent 48 hours.

clopidogrel. Patients without embolic signals were treated with aspirin alone. Patients with atrial fibrillation or another high-risk cardiac source of embolism were treated with anticoagulants. Other patients were treated with aspirin alone.

All patients discharged home were followed up in the clinic with face-to-face interviews by the same neurologist seen at admission within a month from symptom onset. Patients were carefully screened for recurrent vascular events. Early ischemic recurrence was defined as recurrent stroke or TIA within 7 days of symptom onset. The clinical severity of recurrent stroke was evaluated by using the NIHSS.

\section{Statistical Methods}

All analyses were performed with the Statistical Package for the Social Sciences, Version 14.0 software (SPSS, Chicago, Illinois). We used the Student $t$ test for continuous variables and contingency tables with the Fisher exact test for qualitative variables. All tests were 2 -sided. Sensitivity, specificity, positive predictive value, negative predictive value, and unadjusted odds ratios with their $95 \%$ confidence intervals were calculated to evaluate the performance of MR imaging variables in the prediction of early recurrent stroke or TIA. Tested MR imaging variables included the following: $>50 \%$ stenosis or occlusion of the proximal intracranial artery as defined above, $>50 \%$ stenosis or occlusion of the intracranial artery (distal or proximal), and an acute ischemic lesion on DWI. We used logistic regression analysis to adjust for the $\mathrm{ABCD}^{2}$ score. A $P$ value $<.05$ was applied for significance.

\section{RESULTS}

A total of 314 patients were consecutively admitted to the TIA clinic from June 2009 to January 2011. Diagnosis of TIA was ruled out in 81 patients. Eleven additional patients had an isolated amaurosis fugax. Of the 222 remaining patients, 159 were admitted within 24 hours of symptom onset and 129/159 (81.1\%) would have MR imaging within 24 hours of symptom onset. The study population comprised $74(57.3 \%)$ men and 55 (42.6\%) women. Their mean age was $63.5 \pm 15.3$ years.

\section{MRA and DWI Data}

Sixteen (12.4\%) patients had symptomatic significant intracranial artery lesions. Thirteen had grade 1 stenosis, and 3 had occlusion. MRA lesions were classified as proximal in $11(8.5 \%)$ patients and distal in 5 patients. The agreement between both observers in interpreting 3D TOF images was perfect for $96 \%$ of patients. Characteristics of patients and findings on DWI stratified by MRA lesions are summarized in Table 1 . Diabetes was marginally more common in patients with MRA lesions $(P=.07)$. Extracranial carotid stenosis and atrial fibrillation were not associated with MRA lesions. DWI showed acute cerebral infarction in 42 (32.5\%) patients. Most (81.2\%) patients with significant MRA 

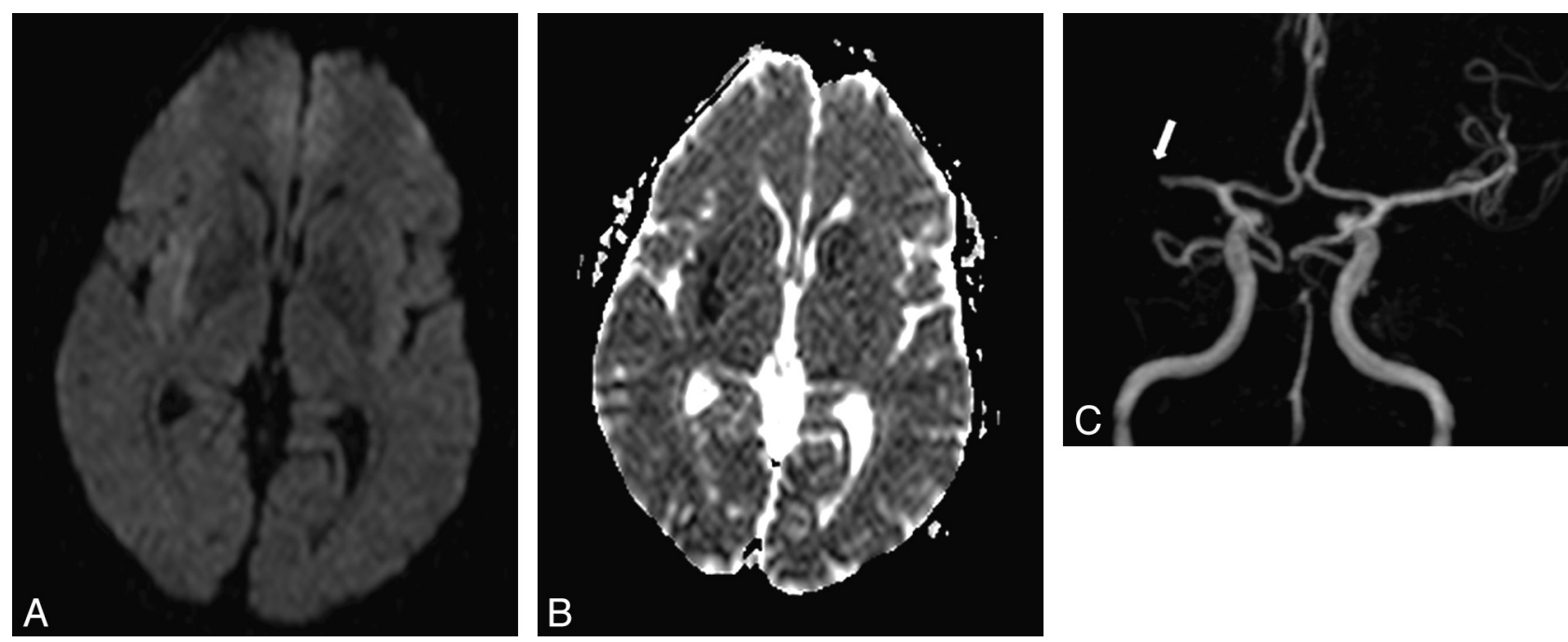

FIG 2. A 59-year-old man with left regressive hemiparesis (clinical duration $=3$ minutes, delay since the first clinical symptom $=3$ hours). $A B C D^{2}$ score $=4$. $A$, DWI demonstrates a subtle hyperintensity in the right subinsular region with corresponding decrease of $A D C$ on the $A D C$ map $(B)$. $C, 3 D$ TOF depicts right M1 segment grade 2 occlusion (arrow), responsible for the acute ischemic lesion in the insular lobe. The patient had another left transient hemiparesis in the subsequent 48 hours.

lesions but only $25.6 \%$ of patients without significant MRA lesions had associated acute infarction on DWI $(P<.001)$.

\section{Clinical Outcome}

There was no recurrent neurologic event during follow-up among patients discharged home. Nine (5\%) patients who were hospitalized according to the predefined criteria for hospitalization had early cerebral ischemic recurrence (7 TIAs, 2 minor strokes, NIHSS score at discharge $=1$ for both). All recurrences occurred within 48 hours of the initial TIA. Individual data from patients with recurrence are summarized in Table 2 . Three of the 9 patients had symptomatic MRA lesions (grade 1 proximal stenosis in 2 patients and proximal occlusion in 1 patient), and 3 patients ( 2 with MRA lesions) had acute infarction on DWI. All recurrences among patients with steno-occlusive lesions on MRA were in the territory of the stenotic/occluded artery. The performance of MR imaging variables as predictors of recurrent TIA or stroke within 7 days is summarized in Table 3. The presence of significant stenosis or occlusion on proximal intracranial vessels at baseline was significantly correlated with early recurrent stroke/TIA $(P=.03)$ with a high specificity (93.3\%) but a low sensitivity (33.3\%) (Figs 1 and 2).The correlation of symptomatic stenosis or occlusion at any site (distal or proximal) with early recurrence was weaker and not statistically significant. DWI did not predict early recurrent stroke/TIA.

Early recurrence was associated with an $\mathrm{ABCD}^{2}$ score of $\geq 4$ $(P=.03)$ but not with extracranial carotid stenosis or atrial fibrillation (Table 4). In multivariate analysis, if one adjusted for the $\mathrm{ABCD}^{2}$ score, a proximal lesion on MRA remained significantly associated with early recurrent stroke/TIA (OR, 5.5; 95\% CI, 1.127.8; $P=.04)$. The rate of early recurrence was $27.3 \%$ in patients with MRA proximal lesions, and among patients without MRA proximal lesions, it was $10.2 \%$ in patients with $\mathrm{ABCD}^{2}$ scores of $\geq 4 \%$ and $0 \%$ in patients with $\mathrm{ABCD}^{2}$ scores of $<4$.
Table 4: Characteristics of patients with early recurrence ${ }^{a}$

\begin{tabular}{|c|c|c|c|}
\hline & $\begin{array}{c}\text { Early } \\
\text { Recurrence } \\
(n=9)\end{array}$ & $\begin{array}{l}\text { No Early } \\
\text { Recurrence } \\
(n=120)\end{array}$ & $\begin{array}{c}P \\
\text { Value }^{b}\end{array}$ \\
\hline Atrial fibrillation & $1(11.1)$ & $8(6.7)$ & 1 \\
\hline Extracranial carotid stenosis $>50 \%$ & $1(11.1)$ & $11(9.2)$ & 1 \\
\hline$A B C D^{2}$ score $\geq 4$ & $8(88.9)$ & $59(49.2)$ & 0.03 \\
\hline
\end{tabular}

${ }^{a}$ Values in parentheses are percentages unless otherwise indicated.

${ }^{\mathrm{b}}$ Fisher exact test.

\section{DISCUSSION}

We have evaluated the performance of TOF-MRA as a predictor of recurrent stroke/TIA at 7 days in patients with TIA. We found that $>50 \%$ stenosis or occlusion of a large proximal intracranial symptomatic artery was significantly associated with recurrent stroke/TIA.

The usefulness of MRA to predict stroke recurrence after TIA has been previously reported by other investigators. Coutts et $\mathrm{al}^{8}$ found a greater risk of recurrent stroke at 90 days among 120 patients with TIA or minor stroke in the presence of an intracranial vessel occlusion on MRA. Our study confirms this finding and further suggests that both stenosis and occlusion of a symptomatic intracranial artery may have an impact on the risk of clinical recurrence. We were able to assess the influence of vascular lesion location and found that only proximal vessel lesions were associated with increased risk of early recurrence. Patients with such vascular lesions were at high $(27.3 \%)$ risk of early recurrence in our study. The sensitivity of MRA was, however, low at $33.3 \%$, suggesting that additional diagnostic features need to be investigated to improve estimates of the risk for early recurrence in individual patients.

DWI was not correlated with the risk of early recurrence in our study. This finding is at variance with those in previous reports, which have consistently demonstrated the value of DWI as a marker for the identification of patients at high risk of early stroke after TIA. ${ }^{6,7,14,15}$ It is possible that the relatively small sample size of our study explains this negative finding. Nevertheless, the pro- 
portion of patients with acute infarction $(32.5 \%)$ was comparable with that reported $(27.6 \%)$ in a large collaborative survey including 4574 patients. ${ }^{6}$ DWI abnormalities were strongly correlated with MRA lesions in our study. The sensitivity of DWI for early recurrence was similar to that of MRA. In contrast, the specificity of DWI was weaker. MRA was thus more accurate than DWI as a predictor of early recurrence after TIA.

The $\mathrm{ABCD}^{2}$ score is a clinical tool designed to predict stroke within 7 days after TIA. The scoring system has been widely implemented to guide triage of patients. Our study confirms that patients with elevated $\mathrm{ABCD}^{2}$ scores have a higher risk of early recurrence. However, MRA remained a significant predictor of early recurrence in a multivariate analysis adjusting for the $\mathrm{ABCD}^{2}$ score. Combining MRA and the $\mathrm{ABCD}^{2}$ score identified all patients with subsequent early recurrence.

Symptomatic extracranial carotid stenosis is an unstable condition associated with early risk of recurrent stroke. ${ }^{16}$ We could not confirm this association in our study, possibly because there were only 12 patients with such arterial lesions. In addition, specific care of these patients, including screening for microembolization, combined antiplatelet therapy, and early carotid endarterectomy, may have reduced the incidence of recurrent stroke.

There are some potential limitations to our study. A relatively long time to admission might have led to a selection bias if some high-risk patients had stroke very early after TIA and therefore were not referred to the TIA clinic. We limited our analysis to patients evaluated within 24 hours of symptom onset to reduce this potential bias.

One-fifth of patients admitted within 24 hours of symptom onset during the study period could not be included in the present analysis because MR imaging was either contraindicated or could not be performed in due time. Timely access to MR imaging is a relevant issue in most communities and several academic centers. Because our findings suggest that MRA might be more accurate than DWI as a predictor of early recurrence, further work should ascertain the predictive value of more accessible vascular imaging modalities such as CT angiography or transcranial color-coded sonography.

The number of recurrences was very low in our study. Thus, although the association of proximal lesions on MRA with early recurrent stroke/TIA was statistically significant, our findings need to be confirmed in a larger number of cases.

The Stroke Outcomes and Neuroimaging of Intracranial Atherosclerosis trial showed that MRA could reliably exclude the presence of 50\%-99\% stenosis of large proximal arteries on angiography. However, the positive predictive value of MRA was not as good. ${ }^{17}$ Similarly, we found that MRA performed better at excluding early recurrence than at identifying it. This may suggest that TOF-MRA is not the best test to identify patients at risk. It is possible that technical improvements, such as using 3T MRA or gadolinium, may improve the positive predictive value of MRA for stroke/TIA recurrence.

We could not determine the nature of MRA lesions. Occlusion could result from embolism or in situ thrombosis. Stenosis is usually attributed to intracranial atherosclerosis but can also result from partial lysis of embolus. The association of MRA lesions with diabetes - a known risk factor for intracranial atherosclero$\operatorname{sis}^{18}$-favors intracranial atherosclerosis as the more likely un- derlying lesion in our study. However, serial examinations would have been necessary to definitely distinguish emboli from intracranial atherosclerosis.

\section{CONCLUSIONS}

The present study highlights intracranial MR imaging features associated with early recurrent stroke/TIA after TIA. Patients with steno-occlusive arterial lesions, specifically proximal lesions, are at high risk of recurrent neurologic events in the 7 days after TIA. Findings suggest that imaging of intracranial arteries should be included in the initial evaluation of TIA to improve triage of patients and tailor secondary prevention.

\section{ACKNOWLEDGMENTS}

We are grateful to D. Seidenwurm, MD, for his valuable editing of the manuscript.

Disclosures: Nathalie Nasr-UNRELATED: Travel/Accommodations/Meeting Expenses Unrelated to Activities Listed: Boehringer Ingelheim, * Sanofi Aventis, ${ }^{*}$ AstraZeneca, ${ }^{*}$ Comments: Boehringer Ingelheim: travel expenses for participation in the European Stroke Conference, which was held in Nice in May 2009; Sanofi Aventis: travel expenses for participation in the Journées de la Société Française de Neurologie Vasculaire, which was held in Paris in November 2010; Astra-Zeneca: travel expenses for participation in the European Stroke Conference, which was held in Hamburg in May 2011. Christophe Cognard-UNRELATED: Consultancy: Consultant for Stryker, Codman, ev3. Vincent Larrue-UNRELATED: Grants/Grants Pending: Sanofi-Aventis, ${ }^{\star}$ Boehringer-Ingelheim, ${ }^{*}$ Payment for Lectures (including service on Speakers Bureaus): Boehringer-Ingelheim, ${ }^{*}$ Travel/Accommodations/Meeting Expenses Unrelated to Activities Listed: Boehringer-Ingelheim.* *Money paid to the institution.

\section{REFERENCES}

1. Giles MF, Rothwell PM. Systematic review and pooled analysis of published and unpublished validations of the ABCD and ABCD2 transient ischemic attack risk scores. Stroke 2010;41:667-73

2. Rothwell PM, Giles MF, Flossmann E, et al. A simple score (ABCD) to identify individuals at high early risk of stroke after transient ischaemic attack. Lancet 2005;366:29-36

3. Johnston SC, Rothwell PM, Nguyen-Huynh MN, et al. Validation and refinement of scores to predict very early stroke risk after transient ischaemic attack. Lancet 2007;369:283-92

4. Lou M, Safdar A, Edlow JA, et al. Can ABCD score predict the need for in-hospital intervention in patients with transient ischemic attacks? Int J Emerg Med 2010;3:75-80

5. Sheehan OC, Kyne L, Kelly LA, et al. Population-based study of ABCD2 score, carotid stenosis, and atrial fibrillation for early stroke prediction after transient ischemic attack: the North Dublin TIA study. Stroke 2010;41:844-50

6. Giles MF, Albers GW, Amarenco P, et al. Addition of brain infarction to the ABCD2 Score (ABCD2I): a collaborative analysis of unpublished data on $\mathbf{4 5 7 4}$ patients. Stroke 2010;41:1907-13

7. Coutts SB, Eliasziw M, Hill MD, et al. An improved scoring system for identifying patients at high early risk of stroke and functional impairment after an acute transient ischemic attack or minor stroke. Int J Stroke 2008;3:3-10

8. Coutts SB, Simon JE, Eliasziw M, et al. Triaging transient ischemic attack and minor stroke patients using acute magnetic resonance imaging. Ann Neurol 2005;57:848-54

9. Korogi $\mathrm{Y}$, Takahashi M, Mabuchi $\mathrm{N}$, et al. Intracranial vascular stenosis and occlusion: diagnostic accuracy of three-dimensional, Fourier transform, time-of-flight MR angiography. Radiology 1994;193:187-93

10. Easton JD, Saver JL, Albers GW, et al. Definition and evaluation of transient ischemic attack: a scientific statement for healthcare professionals from the American Heart Association/American Stroke Association Stroke Council; Council on Cardiovascular Surgery 
and Anesthesia; Council on Cardiovascular Radiology and Intervention; Council on Cardiovascular Nursing; and the Interdisciplinary Council on Peripheral Vascular Disease-the American Academy of Neurology affirms the value of this statement as an educational tool for neurologists. Stroke 2009;40:2276-93

11. Brott T, Adams HP Jr, Olinger CP, et al. Measurements of acute cerebral infarction: a clinical examination scale. Stroke 1989;20:864-70

12. Degnan AJ, Gallagher G, Teng Z, et al. MR angiography and imaging for the evaluation of middle cerebral artery atherosclerotic disease. AJNR Am J Neuroradiol 2011 Sep 22. [Epub ahead of print]

13. Markus HS, Droste DW, Kaps M, et al. Dual antiplatelet therapy with clopidogrel and aspirin in symptomatic carotid stenosis evaluated using Doppler embolic signal detection: the Clopidogrel and Aspirin for Reduction of Emboli in Symptomatic Carotid Stenosis (CARESS) trial. Circulation 2005;111:2233-40
14. Merwick A, Albers GW, Amarenco P, et al. Addition of brain and carotid imaging to the $\operatorname{ABCD}(2)$ score to identify patients at early risk of stroke after transient ischaemic attack: a multicentre observational study. Lancet Neurol 2010;9:1060-69

15. Calvet D, Touze E, Oppenheim C, et al. DWI lesions and TIA etiology improve the prediction of stroke after TIA. Stroke 2009;40: 187-92

16. Ois A, Cuadrado-Godia E, Rodriguez-Campello A, et al. High risk of early neurological recurrence in symptomatic carotid stenosis. Stroke 2009;40:2727-31

17. Feldmann E, Wilterdink JL, Kosinski A, et al. The Stroke Outcomes and Neuroimaging of Intracranial Atherosclerosis (SONIA) trial. Neurology 2007;68:2099-106

18. Bae HJ, Lee J, Park JM, et al. Risk factors of intracranial cerebral atherosclerosis among asymptomatics. Cerebrovasc Dis 2007;24:355-60 